\title{
Sympatric Speciation in Threespine Stickleback: Why Not?
}

\author{
Daniel I. Bolnick \\ Howard Hughes Medical Institute; Section of Integrative Biology, University of Texas at Austin, One University Station C0930, Austin, \\ TX 78712, USA \\ Correspondence should be addressed to Daniel I. Bolnick, danbolnick@mail.utexas.edu \\ Received 3 May 2011; Accepted 25 June 2011 \\ Academic Editor: Andrew Hendry \\ Copyright (C) 2011 Daniel I. Bolnick. This is an open access article distributed under the Creative Commons Attribution License, \\ which permits unrestricted use, distribution, and reproduction in any medium, provided the original work is properly cited.

\begin{abstract}
Numerous theoretical models suggest that sympatric speciation is possible when frequency-dependent interactions such as intraspecific competition drive disruptive selection on a trait that is also subject to assortative mating. Here, I review recent evidence that both conditions are met in lake populations of threespine stickleback (Gasterosteus aculeatus). Nonetheless, sympatric speciation appears to be rare or absent in stickleback. If stickleback qualitatively fit the theoretical requirements for sympatric speciation, why do they not undergo sympatric speciation? I present simulations showing that disruptive selection and assortative mating in stickleback, though present, are too weak to drive speciation. Furthermore, I summarize empirical evidence that disruptive selection in stickleback drives other forms of evolutionary diversification (plasticity, increased trait variance, and sexual dimorphism) instead of speciation. In conclusion, core assumptions of sympatric speciation theory seem to be qualitatively reasonable for stickleback, but speciation may nevertheless fail because of (i) quantitative mismatches with theory and (ii)
\end{abstract} \\ alternative evolutionary outcomes.
}

\section{Introduction}

The feasibility and prevalence of sympatric speciation have been in contention since the birth of evolutionary biology $[1,2]$. Recent empirical work on sympatric speciation has focused on documenting a few likely examples [3-6], or estimating the relative frequency of various geographic modes of speciation [7]. Concurrently, theory has focused on determining which conditions are necessary and sufficient for sympatric speciation to occur [8-11]. Unfortunately, these two related research programs rarely intersect. Namely, there is a need for models based on empirically measurable parameters, preferably tailored to the natural history of specific case studies (e.g., [12, 13]), and for empirical estimates of key parameters in such models. Such fusions of empirical data and theory will provide more biologically realistic insights into when or why sympatric speciation might succeed or fail, and thus explain its frequency.

In this paper, I attempt such a fusion, by comparing simulation results to empirically derived parameter estimates from threespine stickleback (Gasterosteus aculeatus). The goal is to understand why sympatric speciation is rare or absent in this organism [14] despite a qualitative fit with some key requirements for speciation. The objections to sympatric speciation are so well known $[1,2]$ that many biologists take it for granted that sympatric speciation is unlikely. If so, why bother explaining a specific example in which sympatric speciation fails? The answer is that we might find sympatric speciation fails for unexpected reasons.

\section{Theoretical Background}

Theory has revealed two key underpinnings of sympatric speciation. First, a negative frequency-dependent process such as intraspecific competition is required to generate persistent disruptive selection [15-18], which splits the population into phenotypically divergent groups. Second, assortative mating is required to generate reproductive isolation that maintains those divergent groups in the face of recombination $[19,20]$. There is a broad consensus from existing theory that sympatric speciation is easiest when disruptive selection and assortative mating act on the same trait (or closely correlated traits) [11]. Such pleiotropic traits have been dubbed "magic traits" for their uniquely favorable role in speciation [21], because they prevent recombination from decoupling the target of mate choice and selection. 


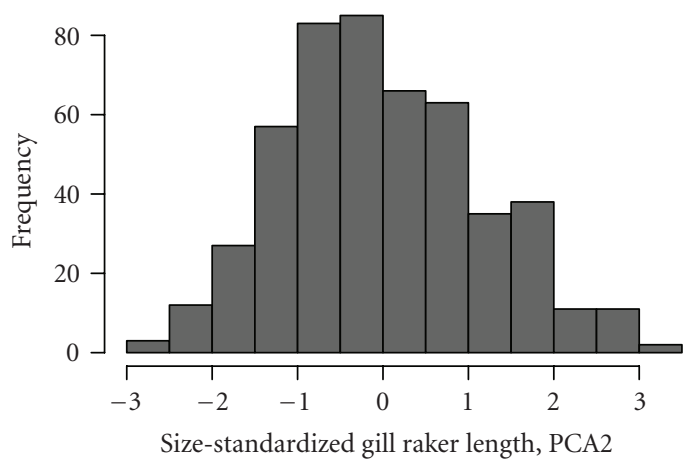

(a)

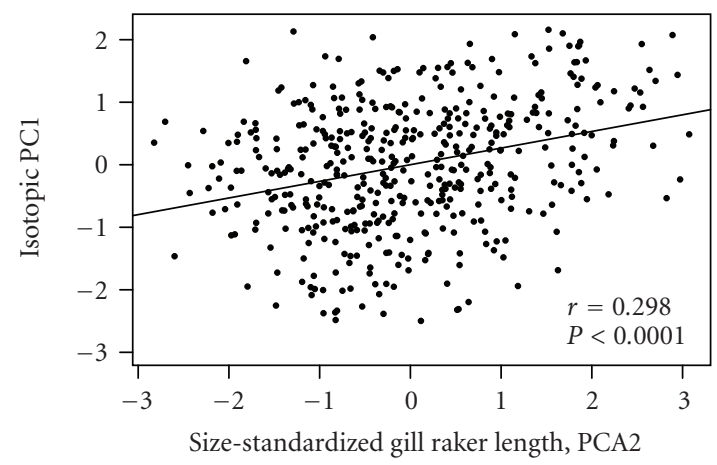

(c)

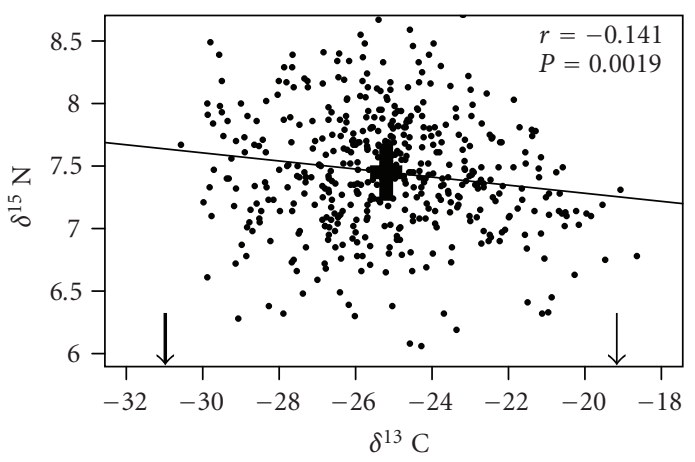

(b)

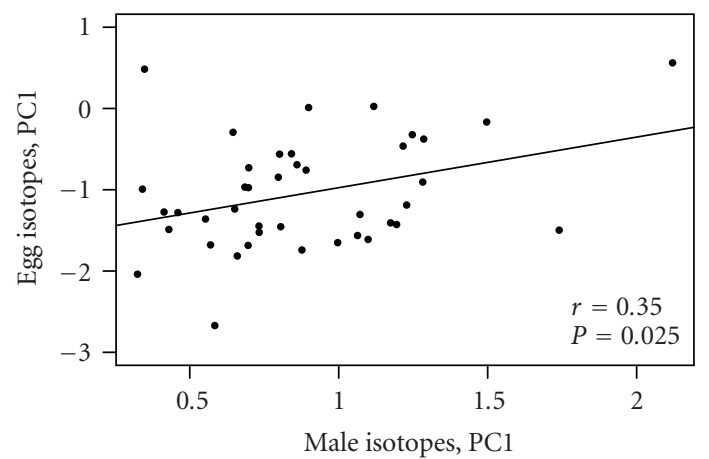

(d)

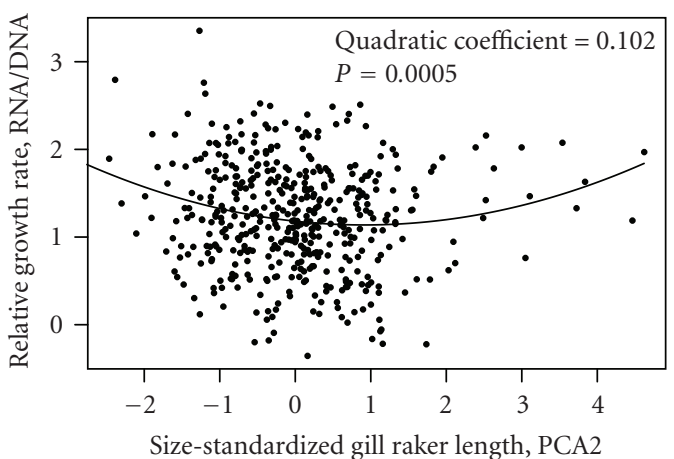

(e)

FIGURE 1: Illustration of the evidence for phenotypic and diet variation, phenotype-diet correlation, disruptive selection, and assortative mating in stickleback. (a) Phenotypic variation in gill raker length within a population of stickleback (First Lake, 2008; [22]). (b) Diet variation within a population of stickleback (First Lake, 2008; [22]) indicated by the high variance in carbon and nitrogen stable isotope ratios $\delta^{13} \mathrm{C}$ and $\delta^{15} \mathrm{~N}$ compared to the variance observed when individuals all consume the same set of prey (thick cross over the centroid represents \pm 2 s.d., based on lab-raised stickleback [23]). Adjusting for baseline isotopic variation (thin arrow indicates a benthic primary consumer [snails], thick arrow represents a planktivorous primary consumer [mussels]), individual stickleback fall anywhere from $0 \%$ to $100 \%$ benthic carbon. Because of the correlation between $\delta^{13} \mathrm{C}$ and $\delta^{15} \mathrm{~N}$, the first isotopic principal component axis can be used to represent the axis from benthic to limnetic diets. (c) Phenotypic and diet variation are correlated: fish with longer gill rakers tend to consume a more limnetic diet (high values of isotopic PC1 indicate lower $\delta^{13} \mathrm{C}$, higher $\delta^{15} \mathrm{~N}$; First Lake). (d) Assortative mating by diet, indicated by a positive correlation between males' isotopic signature and the isotopes from eggs (indicative of female diet; modified from [23]). (e) A key measure of trophic morphology, gill raker length, is subject to disruptive selection as indicated by a significant quadratic coefficient in a regression of growth rate on size-standardized gill raker length. (First Lake, 2005; modified from [24]). Growth rate was measured using a biochemical index, the ratio of RNA to DNA in muscle tissue. The relationships in each panel of this figure are repeatable across multiple solitary lake populations. 
Skeptics of sympatric speciation typically adopt several objections to this theory $[1,11,20,25]$, arguing that (i) models often assume excessively high initial genetic variance and/or high mutation rates, (ii) disruptive selection is likely to be dynamically unstable (populations evolve away from fitness minima towards regions of stabilizing selection), and therefore, disruptive selection should be rare, (iii) costs to mate choice will select against assortative mating, and (iv) "magic traits" subject to both selection and assortment are rare. Finally, casual readers of the literature on speciation theory often come away with the impression that sympatric speciation is all but inevitable when disruptive selection and assortative mating jointly act on a magic trait. In fact, speciation can only occur if these evolutionary forces are strong. Otherwise, the population may evolve higher trait variance, possibly even a bimodal phenotypic distribution with some assortative mating, but never reach full speciation in the sense of forming strongly reproductively isolated and phenotypically nonoverlapping populations [8]. Thus, the core theoretical conflict between skeptics and opponents of sympatric speciation concerns the biological realism of core assumptions, and thus can be best addressed by answering the following empirical questions.

(i) Do Single Populations Typically Exhibit Substantial Genetic Variance in Ecologically Relevant Traits and/or Mating Strategy? There is no doubt that many populations harbor substantial variation for ecologically relevant traits such as resource use. Within many populations, discrete morphotypes or quantitative trait variation give rise to heritable among-individual differences in prey preferences $[26,27]$. As a result, individuals typically consume a small subset of their population's collective diet (across 142 measures of diet variation from 35 species, there was an average of only $45 \%$ similarity between individual and population diets [28]). It is less clear how often populations harbor heritable variation for the degree of choosiness in assortative mating though such variation has been documented [29].

(ii) How Common Is Disruptive Selection? One can measure disruptive and stabilizing selection by quadratic regression of a fitness measure against a phenotype [30]. Positive quadratic coefficients $(\gamma)$ imply disruptive selection if the fitness minimum lies within the range of extant phenotypes. A meta-analysis of selection gradients in nature found that positive and negative quadratic coefficients were about equally common and of similar magnitude (though both were weak: average $|\gamma| \sim 0.1$ [31]). This suggests that disruptive selection is more widespread than often believed, perhaps because it is stabilized by negative frequencydependent interactions that prevent evolution away from fitness valleys towards fitness peaks $[15,32]$. One caveat is that it is not known how many cases of positive curvature entail a real fitness minimum within the phenotypic range, as opposed to just a monotonic but curved fitness function.

(iii) How Common Is Assortative Mating within Populations? Assortative mating is the tendency for individuals to choose mates who are more (or less) phenotypically similar to themselves than expected by chance. The result is a phenotypic (or genetic) correlation between mated males and females, with respect to one or more traits expressed in both sexes. This correlation is easily measured by quantifying some trait in both sexes across multiple mated pairs. The magnitude and sign of this correlation provides a measure of the strength and direction of assortative mating. One caveat is that assortative mating might act on multiple traits concurrently, in which case multivariate canonical correlations might be more appropriate. Studies focusing on one or a few traits might thus underestimate assortative mating by overlooking one or more important traits. With these caveats in mind, a recent meta-analysis established that there is a broad tendency towards weak positive assortative mating within ostensibly panmictic populations (mean $r=0.23$; [33]). Note that these positive correlations can arise from a variety of mate choice processes, including a preference for phenotypically similar mates (as assumed in many sympatric speciation models), spatial sorting of phenotypes at the time of mating, allochrony, or directional mate preferences in both sexes.

(iv) How often Do Disruptive Selection and Assortative Mating Affect the Same Trait? If disruptive selection and positive assortative mating are both moderately common, it is likely that they sometimes act concurrently in a given species, possibly on the same (or correlated) traits. Although examples of "magic traits" have been documented (particularly in incipient species pairs; e.g., $[5,34,35])$, we do not know their general frequency in nature, particularly within populations prior to speciation. Thus, at present, we must rely on insights obtained from specific case studies. For example, disruptive selection and assortative mating have been shown to act on the same traits in Darwin's finches [36, 37] and in stickleback $[23,24]$. In the remainder of this paper, I focus on the latter as a case study.

(v) When They Coincide, Are Disruptive Selection and Assortative Mating Strong Enough to Drive Speciation? To date, this question has remained essentially unanswered for two reasons. First, there are few species for which both processes are known to co-occur within a single population. Second, theoretical models are usually constructed using hard-to-measure parameters, making it hard to evaluate what constitutes (un)realistically strong parameter values.

Threespine stickleback (Gasterosteus aculeatus, a small north-temperate fish) is one of the few organisms known to experience both disruptive selection and assortative mating on a shared trait within single populations (see also studies of Darwin's finches [36, 37]). In the remainder of this paper, I review recent work supporting this claim, focusing on processes occurring in single-species populations of stickleback that represent plausible precursors to speciation (Figure 1(a); as opposed to the well known stickleback species pairs [38]). However, sympatric speciation is rare or absent in stickleback [14], raising the question, "Why has sympatric speciation failed to occur?" To answer this model, 
I combine the reviewed empirical data with a new numerical simulation to show that disruptive selection and assortative mating in stickleback are too weak to drive speciation. Furthermore, I review empirical evidence that fluctuating selection and alternative forms of diversification further inhibit speciation. Thus, many of the common objections to sympatric speciation do not hold in this system, but other quantitative and qualitative hurdles remain, many of which are not widely incorporated into theoretical models.

\section{Diversifying Forces within Lake Stickleback}

3.1. Disruptive Selection. Theory predicts that stable disruptive selection can arise from frequency-dependent interactions such as intraspecific competition for heterogeneous resources [16]. This resource heterogeneity is most pronounced when the population uses two distinct resource types (e.g., benthic and limnetic prey [39], hard and soft seeds $[36,40]$, etc.) but can also apply to multimodal [41] or unimodally varying resources [42]. When co-occurring individuals use divergent subsets of the available resources, the level of competition experienced by a given individual depends on the density of like phenotypes that consume similar resources, rather than the total population density. Phenotypically average individuals experience stronger competition than rare extreme phenotypes, and thus suffer lower relative fitness (disruptive selection). Note that competition is only one of several ecological interactions that can generate the necessary frequency dependence [43]. However, some form of frequency dependence is necessary to stabilize the disruptive selection; for example, a bimodal resource distribution cannot generate sustained disruptive selection by itself, because the population will evolve to specialize on whichever resource type conveys higher fitness. Negativefrequency dependence arises because this transition to specializing on one resource leads to reduced availability of that resource, favoring an evolutionary shift back towards generalist phenotypes that (inefficiently) use both resources.

Here, I focus on competitive disruptive selection, because this is the most widely modeled source of frequency dependence and the best documented in stickleback. For this disruptive selection to occur, a population must exhibit (i) high population density leading to resource limitation, (ii) among-individual variation in use of a diverse set of resources (known as "individual specialization"; [26]), and (iii) a measurable phenotype correlated with this resource use variation, which is thus subject to frequency-dependent competition. In the following paragraphs, I review the existing evidence from stickleback for each of these conditions and the resulting disruptive selection.

(i) Intraspecific Competition. Numerous laboratory studies confirm that food ration strongly affects stickleback growth, female egg production and spawning frequency, and male reproductive success [44-46]. Stickleback kept at approximately natural density in field enclosures reduced benthic invertebrate density by $54 \%$ [47], implying that stickleback exert top-down control of prey density as required for exploitative competition. Accordingly, experimentally elevated stickleback density in field enclosures suppressed prey density, reducing stickleback stomach content mass, growth, and reproductive investment $[48,49]$ and altered growth rates in seminatural ponds [50-52]. These results suggest that intraspecific competition has the potential to significantly impact stickleback. However, further work is required to provide direct proof of density-dependent population regulation in unmanipulated settings.

(ii) Individual Specialization. As a species, stickleback are ecological generalists, consuming a wide diversity of invertebrate prey including crustacean zooplankton, mites, aquatic and terrestrial insects, and even other stickleback. This is often characterized as a bimodal resource base, comprised large benthic macroinvertebrate prey and smaller limnetic zooplankton [38]. In reality, the resource base is more complex and can be described using a continuous distribution of prey body sizes: within solitary stickleback populations, individuals' mean prey size conforms to a unimodal distribution [47]. Individuals also specialize more finely on prey taxa [41], and on microhabitat type [53], than the benthic/limnetic distinction would imply, though, the benthic/limnetic axis remains a convenient and fairly reasonable simplification.

Within a population, any given individual tends to be relatively specialized compared to its population as a whole [54]. For example, direct observation of foraging individuals in Little Mud Lake (Vancouver Island) showed that some individuals consistently directed more than $90 \%$ of their attacks at benthic prey, whereas others used 70\% mid-water prey, or else specialized on surface prey [55]. Accordingly, within a lake stomach contents reveal among-individual diet variation ranging from mostly benthic to mostly limnetic prey [41]. This stomach content variation is corroborated by a longer-term diet measure based on stable isotope variation among individuals, which reveals that co-occurring individuals vary from $0 \%$ to $100 \%$ dietary benthic carbon. This isotopic variability is far greater than we expect when individuals feed on the same prey in the same proportions (Figure 1(b); [54]). We observe this diet heterogeneity even among individuals held in small $\left(10 \mathrm{~m}^{2}\right)$ enclosures that ensure individuals have equal access to the same prey [56]. It is apparent that diet variation reflects among-individual differences in prey preferences and/or acquisition abilities, rather than coarse-grained differences in prey availability due to spatial separation of foraging individuals.

Some of the diet variation is attributed to morphological differences among individuals (Figure 1(c)). Within any given lake, individuals with relatively long gill rakers typically have more pelagic zooplankton in their stomachs and have correspondingly limnetic carbon and nitrogen stable isotope signatures [23, 54, 57-59]. Gill raker number, body size, body shape, jaw lever ratios, hyoid length, and buccal volume have also been found to be correlated with diet variation [41]. Importantly, several of these trophic traits have been shown to exhibit heritable variation [60-63], and thus should be capable of responding to selection. 
(iii) Frequency Dependence. As a result of individual specialization, not all individuals within a population use the same resources. Within a typical population of stickleback any pair of individuals only share an average of $30 \%$ of their prey in common [41]. Because diet is correlated with morphology, this diet overlap is even lower between morphologically divergent individuals: in one lake, diet similarity was twice as high for the most morphologically similar individuals, compared with the most dissimilar individuals [54]. Consequently, the level of competition experienced by an individual depends not on total population density but on the abundance of individuals with overlapping diets (e.g., similar morphology). Intraspecific competition should thus favor individuals with rare feeding strategies over individuals with common strategies who have proportionally more competitors. Direct evidence for such frequency-dependent competition comes from experiments in artificial ponds. Schluter [52] manipulated the relative frequency of benthic and limnetic species pairs and their hybrids and found that a given phenotype experienced reduced growth when it was the more common form. Although the equivalent study has not yet been done within a single-species population of stickleback, it is reasonable to assume that similar processes operate. Within-population frequency dependence may of course be weaker, given the lower phenotypic variance compared with species-pair lakes [38].

The joint occurrence of competition and diet variation should drive frequency-dependent disruptive selection. Accordingly, a survey of multiple solitary populations of stickleback found a widespread tendency towards disruptive selection on two uncorrelated trophic traits (gill raker length and number; [24]). On average, phenotypically intermediate individuals (of either sex) grow more slowly (Figure 1(e)), attain smaller body size and invest proportionally less mass in reproductive tissue than individuals at either extreme of a given trait. The curvature of the estimated fitness landscape averaged $\gamma \sim 0.06$ (Figure 2), somewhat weaker than the average quadratic curvature found in Kingsolver et al.'s meta-analysis [31]. Artificially elevated stickleback density led to stronger-than-average disruptive selection, whereas reduced density eliminated disruptive selection altogether [48]. These results suggest that intraspecific competition generates disruptive selection in many lake populations of stickleback. However, an important caveat is that that none of the fitness metrics (adult growth rate and variation in reproductive tissue mass) represents total lifetime fitness. Consequently, although intermediate phenotypes grow more slowly as adults and invest less mass in gonads, it is possible that additional components of fitness (larval or juvenile growth, survival, mating success, breeding duration, etc.) might overwhelm the component of selection arising from foraging success as measured in the studies cited above. Because lifetime fitness is effectively impossible to track for individual stickleback of known morphology, our best available estimates of selection are likely to be incomplete.

Even taking these selection estimates at face value, disruptive selection is not ubiquitous. Large or small lakes are dominated by single habitat types (limnetic or benthic), whereas intermediate-sized lakes contain a more

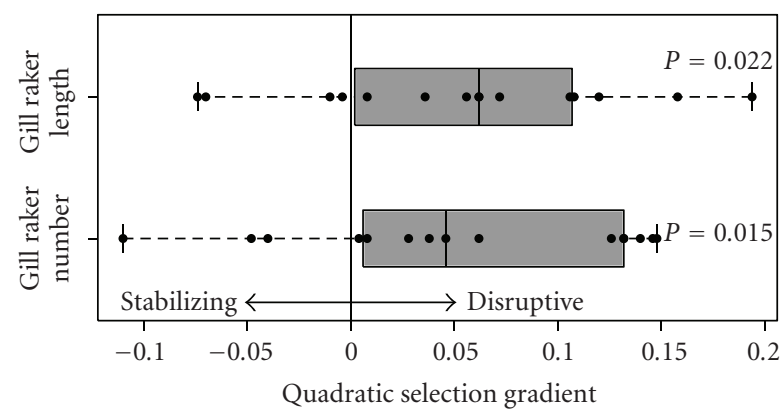

Figure 2: Lake populations exhibit a tendency towards disruptive selection (positive quadratic selection coefficients). Box plots represent the mean (thick vertical lines), 50th quartiles (boxes), and range (whiskers) of quadratic selection gradients for gill raker length and gill raker number from 14 populations, represented as individual dots [24]. $P$ values are provided for $t$-tests of whether the mean quadratic selection gradient is significantly different from zero. The values are modified from [24], who reported the quadratic coefficients $\gamma$ whereas disruptive selection should be measured as $2 \gamma$ as shown here.

equal bination of these resources. Consequently, we expect disruptive selection to be more pronounced in intermediatesized lakes. Indeed, disruptive selection seems to be maximized in intermediate-sized lakes between 40 to 60 ha surface area (there is a significant quadratic relationship between lake area and the quadratic selection coefficient $\gamma$ [24]). Disruptive selection is also modified by other ecological interactions. In experimental ponds, disruptive selection was exaggerated in the presence of predatory trout $[50,64]$. The effect of interspecific competition is not well understood, but depending on the species, fish predators can increase or decrease the diet variation required for disruptive selection [65].

3.2. Assortative Mating. Positive assortative mating occurs when mated pairs are phenotypically more similar than expected by chance. There is abundant evidence that the benthic and limnetic species pairs exhibit premating reproductive isolation [66-70], as do some ecologically and phenotypically divergent allopatric populations [71, 72] though other divergent stickleback populations show no isolation [73]. Remarkably, assortative mating also occurs within solitary stickleback populations. Stable isotope analyses reveal that within a single population, males with more benthic isotope signatures had mated with more benthic females $(r=0.507$; female diet inferred by isotope signature of their eggs; Figure 1(d); [23]). Unlike many studies of reproductive isolation between divergent populations, typically studied in laboratory aquaria, this intraspecific assortment is measured in the field with wild individuals, so it represents a more natural setting. The positive assortative mating represents a "magic trait" system, because individuals pair on the basis of a trait (isotope ratio) which is correlated with the ecomorphological traits under disruptive selection $[23,24]$. In fact, individuals' foraging strategy (reflected in isotopes) 
may be the most direct target of selection, which results in correlated selection on trophic morphology [22].

At present, the mechanism underlying within-population assortative mating in stickleback is unknown. Assortative mating may arise indirectly if different phenotypes are spatially segregated during mating, as has been shown for the benthic and limnetic species pairs [70, 72]. Thus, microhabitat choice could contribute to within-population assortative mating. A spatially explicit follow-up study of isotopic assortative mating [74] (in a second lake population) confirmed the previous positive correlation [23]. Males and females were distributed nonrandomly in space; isotopically more limnetic individuals tended to nest deeper and closer to vegetation than benthic individuals (nests ranged from 0.5 to $2 \mathrm{~m}$ deep) [74]. However, isotopic assortative mating remained even after statistically controlling for spatial and habitat structure, implying a role for active mate preference.

Direct preferences for similar mates might involve morphological traits that are correlated with diet (e.g., size, body shape, and color) or cues derived directly from the prey. Some evidence is available for the latter effect: fish experimentally fed one food type for two weeks, subsequently exhibited a preference to shoal with conspecifics fed the same food [75]. This shoaling preference was maintained as long as the focal individuals received olfactory cues from the potential social groups. Similar results seem to hold when studying females' mate preferences instead of just social affiliation [76]. Olfactory cues have also been implicated in MHC-based mate choice in stickleback $[71,77]$ though it is unclear to what extent this might interact with diet-induced assortment. Regardless of the exact mechanism underlying assortative pairing, the ultimate effect will be to maintain linkage disequilibrium among genes underlying ecologically diverging traits, thus facilitating speciation.

\section{Does Sympatric Speciation Occur in Stickleback?}

4.1. Rarity of Species Pairs. Despite disruptive selection and assortative mating acting on correlated traits, stickleback do not appear to undergo sympatric speciation with any great frequency, if at all [14]. Stickleback occur in north temperate coastal watersheds throughout the Atlantic and Pacific [78]. On Vancouver Island alone, they inhabit many hundreds of lakes that were all likely colonized shortly after deglaciation. Despite this plethora of similar-aged lake populations regionally and globally, only a handful of species pairs have been described. In British Columbia, species pairs have been documented in seven lakes (Balkwill, Enos, Hadley, Paxton, Priest, Emily, and Little Quarry Lakes) in five watersheds from four islands [38, 79, 80]. Collectively, researchers studying stickleback in the American and Canadian northwest have surveyed hundreds of lakes, suggesting that species pairs occur at most in a few percent of inhabited lakes. That said, it is likely that additional species pairs remain to be discovered: a new pair was described as recently as 2008 [79]. Also, it is possible that species pair lakes arise more frequently but commonly collapse [81, 82]. Such a taxon cycle seems unlikely, as the examples of recent species pair collapse entail anthropogenic environmental change $[82,83]$.

A few additional pairs of lacustrine sympatric "morphs" have been described outside of British Columbia [80]. In Alaska, Benka Lake stickleback caught in different habitats are morphologically divergent [84]. However, these ecotypes do not exhibit the bimodal trait distribution seen in species pair lakes, nor is it known whether they are genetically distinct populations. Similar divergence patterns are observed between substrate habitats within several lakes in Iceland [85]. This divergence can evolve rapidly: damming of a fjord in Iceland in 1987 led to rapid divergence from the marine ancestor, and differentiation among habitats [86]. However, these ecomorph pairs do not yet exhibit the near-complete reproductive isolation and genetic divergence that typified the British Columbian species pairs.

4.2. How Did the Few Species Pairs Arise? Not only are species pairs rare, but there is reason to doubt they arose via sympatric speciation. Two alternative models have been proposed for the geography of speciation in British Columbian species pairs $[87,88]$. The first invokes standard sympatric speciation: marine stickleback colonized the postglacial lakes $\sim 12,500$ years ago, after which increased lake elevation due to isostatic rebound prevented recurrent colonization [87]. Subsequently, disruptive selection may have driven ecological divergence, which in turn led to reproductive isolation based on size, color, and behavior. Reproductive isolation may have been a pleiotropic effect of trait divergence [66, 68, 89], supplemented by reinforcement [69].

The alternative model invokes a brief period of allopatry [87]. The initial colonization by marine fish led to rapid adaptation into a generalist that may have resembled today's solitary populations. A subsequent brief sea level rise [90] was thought to have introduced a second population of marine stickleback, which underwent ecological and reproductive character displacement with the native generalist form to produce, respectively, a limnetic and benthic species. This model is neither purely allopatric nor sympatric, since some component of reproductive isolation evolved in both contexts at different times. However, the critical initial step of divergence was allopatric.

For a brief period in the 1990s, phylogeographic data based on mitochondrial DNA sequences lent support to the sympatric model of stickleback speciation, because species pairs for each lake were typically reciprocally monophyletic [88]. However, subsequent analysis of microsatellites told a different story: each ecotype was most closely related to marine forms or to morphs in other lakes [91] though phylogenetic resolution remained poor. The monophyly of mtDNA sequences may thus be the result of introgression following secondary contact. Additional evidence for the allopatric model comes from the fact that limnetic stickleback are more tolerant of salinity [92] and genetically more related to marine forms than are benthic stickleback [91]. Also, all the species pair lakes are geographically nearby and at similar low elevation, suggesting that some 
unique localized geological event facilitated speciation. Consequently, the case for an allopatric phase of divergence seems fairly strong. However, newer geological data does not support the required secondary sea level rise [93], making it hard to explain how a secondary introduction could have happened. Thus, biological data remains most consistent with the secondary contact scenario though the mechanism of secondary colonization is unclear (Schluter, pers. comm.). The question may be unresolvable unless perhaps ancient DNA analyses of subfossil stickleback bones in sediment could reveal evidence for a double invasion.

\section{It Is Not That Easy: Simulations with Empirical Parameters}

The absence (or at best, rarity) of sympatric speciation in lake stickleback despite disruptive selection and assortative mating raises the question, "why do stickleback apparently not experience sympatric speciation?" Several of the standard theoretical objections to sympatric speciation (Section 2, above) do not apply, as there is substantial polymorphism in resource use, disruptive selection, and assortative mating on the ecological trait, as described above. The simplest remaining hypothesis is that disruptive selection and/or assortment, while present, are too weak to complete speciation $[8,94$, 95], or that some additional evolutionary processes impose additional constraints on reproductive isolation.

To test whether the observed selection and assortment are sufficient to drive speciation in a best case scenario, I performed stochastic numerical simulations of the joint effect of disruptive selection and assortative mating acting on a quantitative trait based on the additive effects of numerous small-effect loci (see the Appendix for model details). Unlike most previous models of sympatric speciation, the model used here focused on the effect of empirically measurable parameters (but see the empirically driven papers by Gavrilets and colleagues $[12,13])$. Here, I focus on asking whether the quadratic selection gradients $(\gamma)$ and the phenotypic correlations between mates $(r)$ measured in stickleback are sufficient to split the phenotypically unimodal population into a bimodal distribution of two reproductively isolated populations. This focus on present-day parameter values sets aside the question of whether disruptive selection would drive increased assortative mating in the future. The reinforcement-like process often examined in sympatric speciation models $[8,9,11,96]$ remains empirically untested, and so, it is not studied here explicitly.

Replicate simulations were run for a range of empirically plausible values of disruptive selection and assortative mating to determine what parameter combinations are required for speciation. Speciation was said to have occurred if the initially unimodal phenotype distribution became strongly bimodal, indicated by two major peaks separated by a trough less than $5 \%$ as abundant as the lowest mode. The empirical measures from stickleback (reviewed above) were compared with the simulation results to determine whether solitary stickleback populations could plausibly be expected to speciate sympatrically (e.g., fell within the parameter space

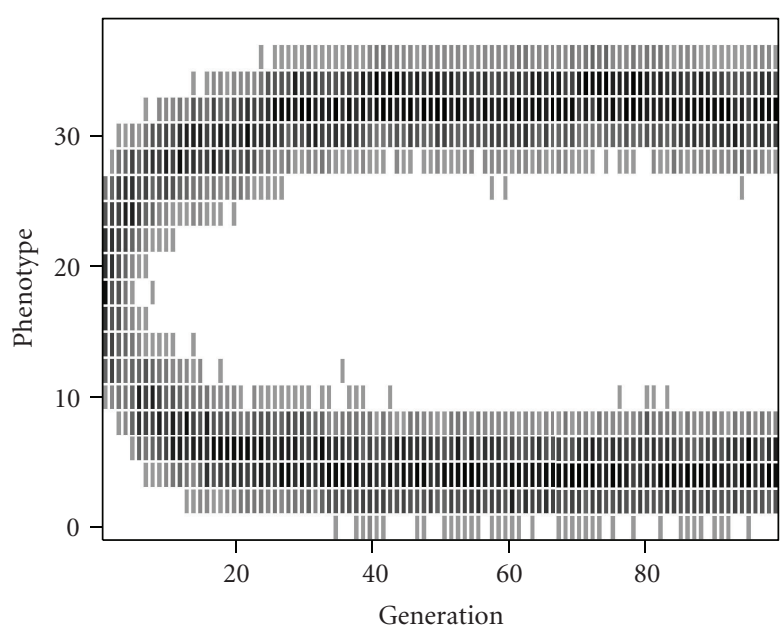

FIGURE 3: An illustration of the simulated dynamics of the phenotype distribution over time, using parameter values that lead to rapid speciation. Darker shading indicates greater numbers of individuals for a given phenotype at a given time. Parameter values are population size $=500$, number of loci $=40$, initial allele frequency $=0.5,2 \gamma=0.4$, and assortative mating correlation $r=$ 0.7 .

conducive to speciation). Note that my simulations focused on just these parameters and omits extensive consideration of other potentially relevant factors such as the genetic architecture of the trait, population size, mutation rate, and costs to choosiness.

Consistent with many previous theoretical models and simulations [97], I found that sympatric speciation was possible given the joint action of strong disruptive selection and assortative mating (Figure 3). By "strong" I mean quadratic selection coefficients of $\gamma>0.25$; empirically, $\gamma$ follows a double exponential distribution and ranges between about -1 to 1 , with most values between -0.1 to 0.1 [31]. Speciation required strong positive assortative mating, defined here as a trait correlation above about 0.6; empirically $r$ ranges from below -0.9 to above 0.9 , with a mean of 0.23 [33]. However, if either evolutionary force is weak or moderate (e.g., average values of $\gamma \sim 0.1$ and $r \sim 0.25$ ), then speciation does not occur (Figure 4). Instead, populations may either remain unimodal but with inflated variance (compare Figure 5(a) with 5(b)), or may attain a uniform or even bimodal distribution (Figure 5(c)) in which assortative mating is too weak to prevent the continual and frequent recreation of intermediate phenotypes $[8,94,98]$ but maintains some linkage disequilibrium that substantially inflates trait variance. Notably, however, sympatric speciation (Figure 5(d)) can be expected to occur with empirically reasonable (albeit strong) parameter values.

Empirically estimated values of disruptive selection and assortative mating in stickleback fall well within the parameter space where speciation is never observed (Figure 4). The strongest observed disruptive selection would be sufficient for speciation, if assortative mating were twice as strong as the observed average. The strongest observed assortative mating would be sufficient if disruptive selection were seven 


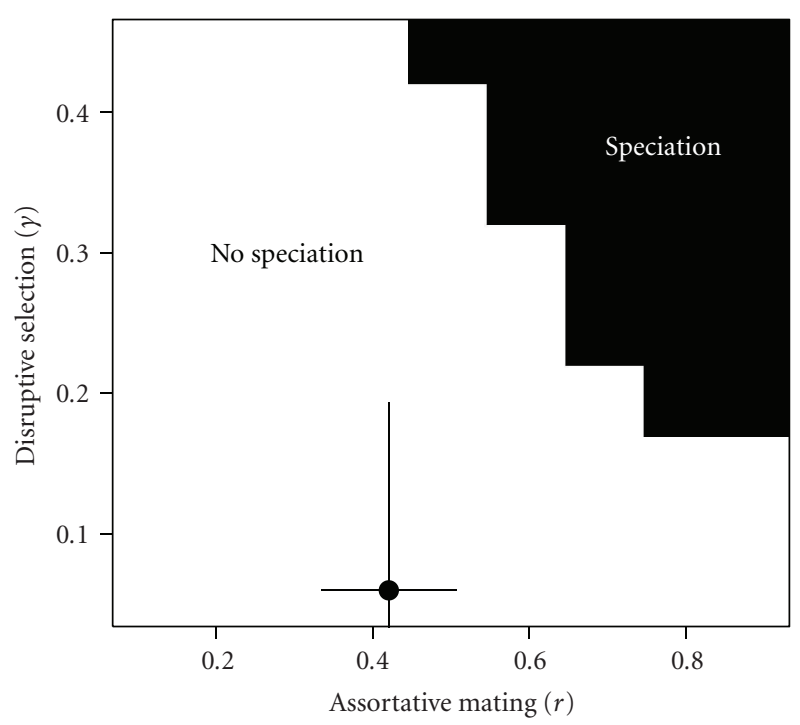

FIgURE 4: Speciation is only observed when there is strong disruptive selection and strong assortative mating (shaded region). For comparison, the crossed lines indicates the range and mean (intersection) of these parameters measured in single-species lake populations of stickleback $[23,24]$. Parameter values are population size $=500$, number of loci $=40$, and initial allele frequency $=0.5$. Disruptive selection and assortative mating were varied systematically from $\gamma=0$ to 0.5 and $r=0$ to 0.95 , in increments of 0.025 . This parameter space falls well within empirically justifiable values based on meta-analyses $[31,33]$. Ten replicate simulations were run for each factorial combination of parameters. Dark shading indicates regions of parameter space in which speciation was observed at least once.

times stronger than average (or more than twice the maximum). Even the joint effect of the empirical maximum disruptive selection and assortative mating would be insufficient for sympatric speciation (in simulations run for $>50,000$ generations). It is thus clear that solitary populations of stickleback are unlikely to undergo sympatric speciation in their current ecological setting. This is a reassuring result in that it may explain why solitary populations have remained solitary. Of course, this does not rule out the possibility that the few stickleback species pairs arose from populations with exceptionally high levels of one or both parameters.

One caveat regarding these simulation results is that I omit the possibility that assortative mating itself may evolve. Numerous models suggest that disruptive selection can drive a reinforcement-like process favoring the evolution of increased assortative mating $[9,43]$ though this possibility remains contentious $[25,99]$. Thus, it is conceivable that stickleback populations experiencing the strongest levels of disruptive selection could evolve stronger mate preferences in the future. This increased choosiness depends on a variety of additional assumptions about the behavioral basis of assortative mating, the genetic architecture of mate choice, costs to mate choice, mating system, and so on. Lacking information about these parameters, it seems more appropriate at present to evaluate whether existing parameter values are sufficient to bifurcate populations, than to speculate over whether

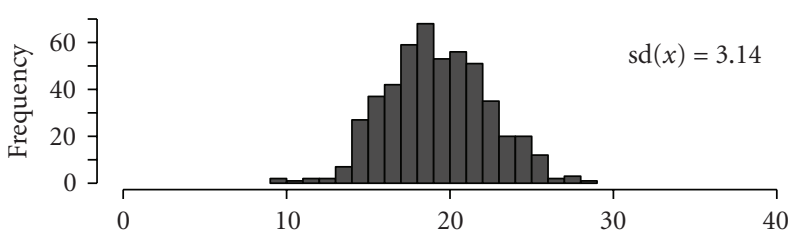

(a)

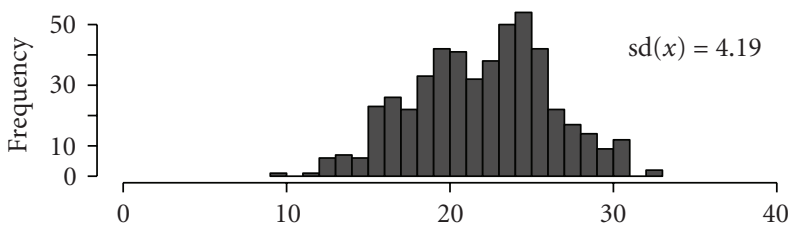

(b)

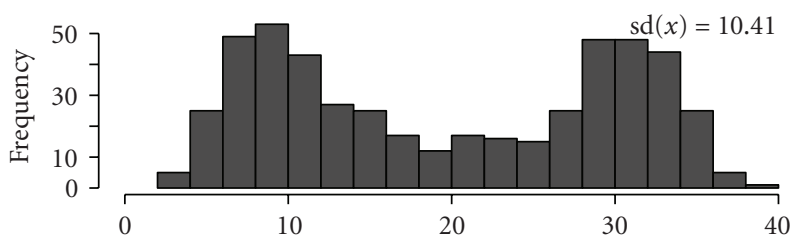

(c)

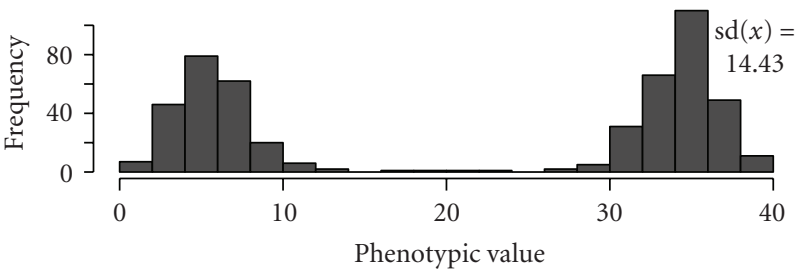

(d)

FIGURE 5: An illustration of various outcomes of disruptive selection and assortative mating, depending on parameter values. Each histogram represents the phenotype frequency distribution at a quasiequilibrium after 1,000 generations. Longer simulation runs do not qualitatively change the phenotype distribution for a given parameter combination. (a) With no disruptive selection and no assortative mating $(\gamma=0, r=0)$, phenotypic variance follows a binomial (approximately normal) distribution whose variance depends on the level of polymorphism at the 40 additive loci, maintained by mutation-drift balance. (b) Using a combination of parameters close to the strongest disruptive selection and assortment observed in stickleback $(2 \gamma=0.15, r=0.5)$, the population attains modestly higher phenotypic variance than in (a) but remains unimodal. (c) Larger parameter values $(2 \gamma=0.3$, $r=0.6$ ) rapidly lead to a weakly bimodal phenotype distribution that does not resolve itself into separate species even after 50,000 generations. (d) Speciation can occur when disruptive selection and assortment are near the upper end of plausible empirical values $(2 \gamma=0.4, r=0.8)$.

stickleback may or may not shift to elsewhere in parameter space. Although I do not explicitly model the evolution of assortment, it is possible to infer the result of a hypothetical increase in female choice, which amounts to moving horizontally right in the parameter space shown in Figure 4. For populations at the highest level of disruptive selection (Figure 4), such a rightward shift in parameter space could conceivably bring them into parameter space where 
speciation is possible. However, for the majority of stickleback populations such reinforced assortative mating would still not facilitate speciation. An important subject for future empirical investigation is whether disruptive selection really does drive the evolution of increased assortative mating.

\section{It Is Not That Easy: Additional Constraints on Speciation in Stickleback}

If reinforcement could push the most disruptive populations into speciation-prone parameter space, one might ask why this has not actually happened. The answer is that there may be additional constraints on sympatric speciation, many of which are not typically considered in speciation models. For example, increased assortment may be inhibited if selection is variable through time, or if the population evolves an alternative solution to disruptive selection. In the last section of this paper, I summarize evidence for both kinds of speciation-inhibiting factors in stickleback.

6.1. Stability of the Fitness Landscape. Theoretical models of speciation typically assume populations are subject to persistent disruptive selection despite general evidence for temporal fluctuations in fitness landscapes [100, 101] (though some fluctuations can be due to sampling noise). Of the 14 lakes studied by Bolnick and Lau [24], First and McNair Lakes were among the ones with the strongest disruptive selection (Figure 2). A second sample of these two lakes, two years later, found significant stabilizing selection in First Lake and directional selection in McNair [22]. The reason for this interannual variation is not clear. One hypothesis is that fluctuating population density [102] (or resource availability) alters the strength of competition and thus the fitness landscape [48]. For instance, a long-term study of a population of Eurasian Perch (Perca fluviatilis) found shifts in the fitness landscape associated with twoorder-of-magnitude population density cycles. At high density, perch experienced disruptive selection, which changed to stabilizing or directional selection during periods of low density [103]. Changes in selection gradients might also reflect interannual variation in predation regimes [50, 104-107] or the strength of interspecific competition [65]. Such variable selection regimes would undermine sympatric speciation $[108,109]$ and might instead favor the evolution of phenotypic plasticity [110]. In fact, temporal changes in the fitness landscape may explain the introgressive collapse of formerly distinct species pairs of stickleback [83].

6.2. Alternative Forms of Diversification. Frequency-dependent competition gives rise to disruptive selection, because the resource distribution can support a broader phenotypic variance than presently found in the population [111-113]. Evolution will thus favor any of a number of evolutionary changes that increase the population's phenotypic variance; assortative mating is just one of several mechanisms that can increase this variance [114]. If some other form of diversification evolves first and mitigates the disruptive selection, sympatric speciation will be less likely [115].
The most straightforward outcome of disruptive selection is increased genetic variance within a phenotypically unimodal population (Figure 5(b)). Consistent with this outcome, derived lacustrine populations of stickleback exhibit higher phenotypic variance (particularly for gill raker traits under disruptive selection) compared to marine stickleback that represent a putative ancestral character state [116]. It is important to note, however, that these results reflect phenotypic variances, and it is not known to what extent among-population differences in phenotypic variances are matched by underlying differences in genetic variances.

Disruptive selection due to intraspecific competition can also drive the evolution of ecological sexual dimorphism $[115,117,118]$, which can inflate trait variance. This intraspecific ecological character displacement occurs under the same theoretical conditions as sympatric speciation [115]. However, dimorphism can evolve more quickly than assortative mating, reducing disruptive selection to the point where sympatric speciation is inhibited [115] though this antagonism can be relaxed if disruptive selection acts along multiple trait axes [119]. Stickleback exhibit sexual dimorphism with respect to diet and associated trophic morphology such as gill raker length $[24,104,120,121]$. This dimorphism is maximized in intermediate sized lakes where disruptive selection is typically strongest [24], supporting the notion that disruptive selection leads to dimorphism [115, 119]. However, controlling for lake size, there is a negative relationship between present-day disruptive selection and dimorphism [24], implying that once dimorphism evolves the fitness landscape becomes flatter, which may inhibit speciation [115]. These results seem to confirm the notion that sexual dimorphism can evolve quickly and mitigate disruptive selection, thereby potentially reducing the capacity for sympatric speciation.

Another possibility is that disruptive selection favors the evolution of phenotypic plasticity [122], allowing individuals to shift onto underused resources within a single generation rather than over evolutionary time [110]. Optimal foraging theory (OFT) is predicated on the idea that individuals are able to flexibly choose prey to maximize their expected fitness [123], accepting lower-value resources as preferred prey become scarce [124]. Applied to phenotypically variable populations, OFT predicts that strong competition might lead to behavioral rather than evolutionary diversification [125]. Consistent with this prediction, experimentally elevated stickleback densities led to greater population niche width and greater among-individual diet variation over the space of a mere two weeks $[41,56]$. Thus, behavioral plasticity clearly does provide a mechanism for diversification in response to changing fitness landscapes. Morphological plasticity may play a similar role albeit over a slower time scale as plasticity relies on somatic growth and reshaping. Stickleback do exhibit diet-induced plasticity, which is greatest in solitary populations (Svanbäck and Schluter, unpublished).

To summarize, stickleback concurrently exhibit several forms of within-population diversification: increased phenotypic variance, dimorphism, behavioral plasticity, and 
morphological plasticity. Any or all of these may increase trophic diversity in ways that mitigate disruptive selection and can inhibit speciation.

\section{Conclusions}

The past two decades have seen a burst of renewed research on sympatric speciation. Collectively, this body of literature suggests that sympatric speciation (1) probably has occurred in at least a few cases $[3,5,6]$ but is still relatively rare $[97,126]$ and difficult to prove and (2) is "theoretically plausible" [127] but requires very specific conditions [25]. An important next step is to fuse the theoretical and empirical work (e.g., $[12,13])$, by empirically evaluating the biological plausibility of the key theoretical assumptions in particular model organisms [97]. This shifts empirical focus away from putative cases of sympatric speciation to a more general program of jointly measuring key parameters (e.g., selection strength, spatial structure and gene flow, strength of assortative mating, and identifying phenotypic targets of mate choice). These estimates can then be used to build a more biologically informed body of theory (e.g., $[12,13])$ or to identify those populations best suited to the various forms of sympatric diversification.

In stickleback, the good news for theoreticians is that the joint occurrence of (weak) disruptive selection and assortative mating validates long-standing assumptions. Metaanalyses suggest that both phenomena are quite common $[31,33]$, though weak and perhaps temporally variable $[100,101]$. While the joint action of disruptive selection and assortment is less well established, the work reviewed above indicates that the processes do co-occur in lake populations of stickleback. The bad news for theory is that both forces are typically too weak to drive speciation. As is often the case in ecological and evolutionary theory, model predictions are sensitive to the precise parameter values used. Consequently, rigorous tests of theory requires models framed using measurable parameters coupled with suitable empirical data. Theoretical models of speciation also typically ignore temporal fluctuations in selection, and alternative forms of diversification, such as we see in stickleback. Such constraints do not make the theory wrong so much as incomplete, echoing Gavrilets" statement that "it is not that easy" [25].

The absence of sympatric speciation in stickleback is not necessarily an indictment of sympatric speciation theory. Indeed, it is the comparison of theory with empirical parameter values that helps illuminate why speciation may not readily occur. Furthermore, existing models have made a variety of related testable predictions, such as the links between competition, habitat diversity, and sexual dimorphism, which have been empirically tested $[24,48,56,65$, 119]. Thus, models of sympatric speciation may be an illustration of G. E. P. Box's statement that, "all models are wrong, but some are useful" [128]. Perhaps continued controversy over sympatric speciation will prove useful as well, regardless of its prevalence in nature, particularly if the debate spurs more extensive empirical measurement of generally important evolutionary forces.

\section{Appendix}

\section{Simulation of Phenotypic Evolution under Disruptive Selection and Assortative Mating}

I used stochastic simulations of an individual-based model to explore the potential for sympatric speciation across a range of magnitudes of disruptive selection and assortative mating. Sympatric speciation has been widely explored with both stochastic and numerical simulations and analytical theory (summarized in [97]), but these models tend not to be framed in terms of empirically measurable parameters. I, therefore, focus here on describing a model that can be framed in terms of disruptive selection gradients and malefemale trait correlations.

I consider a population of $\mathrm{N}$ haploid individuals characterized by a phenotype subject to both disruptive selection and assortative mating. Each individual's phenotype is a quantitative trait that depends on the additive effect of 40 independently assorting loci of small and equal effect. Initially, each locus is assumed to be polymorphic with equal frequencies of two alleles of effect size 0 or 1 . Thus, the phenotype space ranges from a minimum of 0 to a maximum of 40 with an initial mean of 20 and variance 10. Individuals were created by randomly drawing a set of 40 allele values. The general results described here are qualitatively robust to using smaller numbers of loci [63]. The traits under disruptive selection in stickleback are known to be polygenic, with multiple QTL none of which have substantial effect sizes [63]. Consequently, using a moderate to large number of loci is probably appropriate.

In most sympatric speciation models, disruptive selection is an emergent outcome of some assumed ecological process such as intraspecific competition [129]. This adds a degree of realism, to the extent that the ecological process is biologically justified, but makes it difficult to directly control the strength of disruptive selection as measured empirically. Here, I ignored the ecological mechanism underlying selection and instead used a specified disruptive selection gradient to calculate the corresponding fitness for each phenotype in the population. Specifically, I first standardized the population's phenotype distribution to a standard normal $($ mean $=0$, standard deviation $=1)$. Next, individuals' fitness is calculated as

$$
w(x)=w_{\text {center }}+\beta x+\gamma x^{2},
$$

where $x$ is an individual's standardized phenotype, $w_{\text {center }}$ is the fitness of the average phenotype, $\beta$ is the linear selection gradient (assumed to be 0 ), and $\gamma$ is the quadratic selection gradient. Fitness was then standardized by dividing every individuals' fitness $w(x)$ by the maximal fitness, yielding a value that falls within the range 0 to 1 . This fitness is used as the probability the individual survives viability selection.

This selection scheme ensures that the minimum of disruptive selection tracks the phenotype mean, preventing the population from evolving away from the fitness minimum. The stabilized fitness minimum roughly mimics the behavior of models of frequency-dependent competition that generate stable fitness minima required for speciation [15]. Finally, 
a uniform random number was drawn for each individual; if the random number was less an individual's $w(x)$, the individual was retained for mating.

Following a round of selection, the surviving individuals select mates and reproduce. Individuals are randomly assigned a sex assuming an equal sex ratio. One female and one male are randomly drawn from the pool of available survivors, and we calculated the probability the individuals mate based on their phenotypic similarity (see next paragraph for details). If a random uniform number fell below this probability, they produced one offspring and were returned to the pool of breeders. If not, they were simply returned to the breeders, and a new pair was randomly selected. Whether or not a pair mated, they were, therefore, able to subsequently remate. This scheme allows for some sexual selection to occur; for instance, with strong positive assortative mating, rare phenotypes may be unable to find acceptable mates. This mate pairing was iterated until the desired population size of offspring was reached (allowing a constant population size throughout the simulations), at which point all adults died and a new generation began.

The typical speciation model calculates a probability the female accepts the male, given their phenotypic difference

$$
P(\text { mating })=\exp \left[-\frac{\left(x_{\text {male }}-x_{\text {female }}\right)^{2}}{\sigma_{a}^{2}}\right],
$$

where $x$ is the phenotypic value of the male and female being evaluated and $\sigma_{a}^{2}$ is the variance of the mate probability function. This variance measures how quickly mating probability drops off with phenotypic difference. Most speciation models either used a set value of $\sigma_{a}^{2}$ or allow $\sigma_{a}^{2}$ to evolve as a female phenotype (choosiness; e.g., [9]). However, this parameter is difficult to measure empirically in the field. Instead, I ran simulations with a specified male-female correlation, $r$, and used a known relationship to convert $r$ to $\sigma_{a}^{2}$ which we subsequently used in (A.2) to calculate the mating probability for a given male-female pair. Assuming a Lande-style relative mate choice function [130], the variance of the mate choice function and the phenotypic standard deviation determine the resulting correlation between mated pairs

$$
r=\frac{\sigma_{m} \sigma_{f}}{\sqrt{\sigma_{a}^{4}+\sigma_{m}^{2} \sigma_{f}^{2}}}
$$

See [131]. This can be rearranged to calculate a value for $\sigma_{a}^{2}$ given the phenotypic variance for a trait found in both sexes $\left(\sigma_{x}=\sigma_{m}=\sigma_{f}\right)$ and a desired $r$

$$
\sigma_{a}=\left[\frac{\sigma_{x}^{4}}{r^{2}}-\sigma_{x}^{4}\right]^{0.25} .
$$

When individuals did mate, I generated one offspring whose genotype is a random sample of alleles from the two parents. That is, for each locus the offspring receives an allele randomly from one parent. To maintain genetic variation, I allowed a mutation process that inverts the phenotypic value of the inherited allele ( 0 to 1 , or 1 to 0 ) with a specified probability (here, 0.0001, which was chosen because it maintains fairly constant phenotypic variance in the face of drift in simulations without selection or assortment).

I iterated this life cycle (selection, then rounds of mating until the population size was met) for a specified number of generations. At each time step, I recorded the phenotype distribution. At the end of the simulation, speciation was said to have occurred if the phenotype distribution was bimodal, and intermediate phenotypes had a frequency less than $5 \%$ that of the lower of the two modes. I iterated through a range of parameter space ( $\gamma$ from 0 to 0.5 in increments of 0.5 , and $r$ from 0 to 0.95 in increments of 0.5 ). For each parameter combination, I replicated a 1000 generation simulation 10 times and recorded whether or not speciation occurred. I also recorded the phenotypic variance of the ending trait distribution. Simulations were conducted in R [132], and the code is available from the author on request.

\section{Acknowledgments}

The authors would like to thank Chad Brock, Edgar DuenezGuzman, Andrew Hendry, Jonathan Losos, Dolph Schluter, Mike Singer, Michael Turelli, and anonymous reviewers for discussions and feedback on the paper. This work was supported by funding from the David and Lucille Packard Foundation, and many of the reviewed results arose from NSF Grant no. DEB-0412802.

\section{References}

[1] J. A. Coyne and H. A. Orr, Speciation, Sinauer Associates, Sunderland, Mass, USA, 2004

[2] E. Mayr, Evolution and the Diversity of Life, Belknap Press, Cambridge, Mass, USA, 1976.

[3] M. Barluenga, K. N. Stölting, W. Salzburger, M. Muschick, and A. Meyer, "Sympatric speciation in Nicaraguan crater lake cichlid fish," Nature, vol. 439, no. 7077, pp. 719-723, 2006.

[4] P. G. Ryan, P. Bloomer, C. L. Moloney, T. J. Grant, and W. Delport, "Ecological speciation in South Atlantic island finches," Science, vol. 315, no. 5817, pp. 1420-1423, 2007.

[5] V. Savolainen, M. C. Anstett, C. Lexer et al., "Sympatric speciation in palms on an oceanic island," Nature, vol. 441, no. 7090, pp. 210-213, 2006.

[6] U. K. Schliewen, D. Tautz, and S. Paabo, "Sympatric speciation suggested by monophyly of crater lake cichlids," Nature, vol. 368, no. 6472, pp. 629-632, 1994.

[7] T. G. Barraclough and A. P. Vogler, "Detecting the geographical pattern of speciation from species-level phylogenies," American Naturalist, vol. 155, no. 4, pp. 419-434, 2000.

[8] R. Bürger, K. A. Schneider, and M. Willensdorfer, "The conditions for speciation through intraspecific competition," Evolution, vol. 60, no. 11, pp. 2185-2206, 2006.

[9] U. Dieckmann and M. Doebeli, "On the origin of species by sympatric speciation," Nature, vol. 400, no. 6742, pp. 354357, 1999.

[10] U. Dieckmann, M. Doebeli, J. A. J. Metz, and D. Tautz, Eds., Adaptive Speciation, Cambridge University Press, New York, NY, USA, 2004.

[11] S. Gavrilets, "Perspective: models of speciation-what have we learned in 40 years?” Evolution, vol. 57, no. 10, pp. 21972215, 2003. 
[12] S. Gavrilets and A. Vose, "Case studies and mathematical models of ecological speciation. 2. Palms on an oceanic island," Molecular Ecology, vol. 16, no. 14, pp. 2910-2921, 2007.

[13] S. Gavrilets, A. Vose, M. Barluenga, W. Salzburger, and A. Meyer, "Case studies and mathematical models of ecological speciation. 1. Cichlids in a crater lake," Molecular Ecology, vol. 16, no. 14, pp. 2893-2909, 2007.

[14] A. P. Hendry, D. I. Bolnick, D. Berner, and C. L. Peichel, "Forth and back on the speciation continuum: explorations with stickleback," Journal of Fish Biology, vol. 75, no. 8, pp. 2000-2036, 2009.

[15] P. A. Abrams, H. Matsuda, and Y. Harada, "Evolutionarily unstable fitness maxima and stable fitness minima of continuous traits," Evolutionary Ecology, vol. 7, no. 5, pp. 465-487, 1993.

[16] R. Bürger and A. Gimelfarb, "The effects of intraspecific competition and stabilizing selection on a polygenic trait," Genetics, vol. 167, no. 3, pp. 1425-1443, 2004.

[17] M. Doebeli, "A quantitative genetic competition model for sympatric speciation," Journal of Evolutionary Biology, vol. 9, no. 6, pp. 893-909, 1996.

[18] M. L. Rosenzweig, "Competitive speciation," Biological Journal of the Linnean Society, vol. 10, no. 3, pp. 275-289, 1978.

[19] R. Bürger and K. A. Schneider, "Intraspecific competitive divergence and convergence under assortative mating," American Naturalist, vol. 167, no. 2, pp. 190-205, 2006.

[20] J. Felsenstein, "Skepticism towards Santa Rosalia, or why are there so few kinds of animals," Evolution, vol. 35, pp. 124$138,1981$.

[21] S. Gavrilets, Fitness Landscapes and the Origin of Species, Princeton University Press, Princeton, NJ, USA, 2004.

[22] M. Araújo and D. I. Bolnick, "Diet as a primary target for natural selection in the threespine stickleback," Evolutionary Ecology Research. In press.

[23] L. K. Snowberg and D. I. Bolnick, "Assortative mating by diet in a phenotypically unimodal but ecologically variable population of stickleback," American Naturalist, vol. 172, no. 5, pp. 733-739, 2008.

[24] D. I. Bolnick and L. L. On, "Predictable patterns of disruptive selection in stickleback in postglacial lakes," American Naturalist, vol. 172, no. 1, pp. 1-11, 2008.

[25] S. Gavrilets, "'Adaptive speciation"-it is not that easy: a reply to Doebeli et al," Evolution, vol. 59, no. 3, pp. 696-699, 2005.

[26] D. I. Bolnick, R. Svanbäck, J. A. Fordyce et al., "The ecology of individuals: Incidence and implications of individual specialization," American Naturalist, vol. 161, no. 1, pp. 1-28, 2003.

[27] T. B. Smith and S. Skúlason, "Evolutionary significance of resource polymorphisms in fishes, amphibians, and birds," Annual Review of Ecology and Systematics, vol. 27, pp. 111133, 1996.

[28] M. A. Áraujo, C. Layman, and D. I. Bolnick, "The ecological causes of individual specialization," Ecology Letters, vol. 14, pp. 948-958, 2011.

[29] R. K. Butlin, "The variability of mating signals and preferences in the brown planthopper, Nilaparvata lugens(Homoptera: Delphacidae)," Journal of Insect Behavior, vol. 6, no. 2, pp. 125-140, 1993.

[30] R. Lande and S. J. Arnold, "The measurement of selection on correlated characters," Evolution, vol. 37, pp. 1210-1226, 1983.
[31] J. G. Kingsolver, H. E. Hoekstra, J. M. Hoekstra et al., "The strength of phenotypic selection in natural populations," American Naturalist, vol. 157, no. 3, pp. 245-261, 2001.

[32] D. S. Wilson and M. Turelli, "Stable underdominance and the evolutionary invasion of empty niches," American Naturalist, vol. 127, no. 6, pp. 835-850, 1986.

[33] Y. Jiang, D. I. Bolnick, and M. Kirkpatrick, "Widespread positive assortative mating within animal populations," In revision.

[34] O. Puebla, E. Bermingham, F. Guichard, and E. Whiteman, "Colour pattern as a single trait driving speciation in Hypoplectrus coral reef fishes?" Proceedings of the Royal Society B, vol. 274, no. 1615, pp. 1265-1271, 2007.

[35] R. G. Reynolds and B. M. Fitzpatrick, "Assortative mating in poison-dart frogs based on an ecologically important trait," Evolution, vol. 61, no. 9, pp. 2253-2259, 2007.

[36] A. P. Hendry, S. K. Huber, L. F. De León, A. Herrel, and J. Podos, "Disruptive selection in a bimodal population of Darwin's finches," Proceedings of the Royal Society B, vol. 276, no. 1657 , pp. 753-759, 2008.

[37] S. K. Huber, L. F. De Leon, A. P. Hendry, E. Bermingham, and J. Podos, "Reproductive isolation between sympatric morphs in a bimodal population of Darwin's finches," Proceedings of the Royal Society of London B, vol. 274, pp. 1709-1714, 2007.

[38] D. Schluter and J. D. McPhail, "Ecological character displacement and speciation in sticklebacks," American Naturalist, vol. 140, no. 1, pp. 85-108, 1992.

[39] B. W. Robinson and D. S. Wilson, "Character release and displacement in fishes: a neglected literature," American Naturalist, vol. 144, no. 4, pp. 596-627, 1994.

[40] T. B. Smith, "Bill size polymorphism and intraspecific niche utilization in an African finch," Nature, vol. 329, no. 6141, pp. 717-719, 1987.

[41] M. S. Araújo, J. P. R. Guimaraes, R. Svanbäck, A. Pinheiro, S. F. dos Reis, and D. I. Bolnick, "Network analysis reveals contrasting effects of intraspecific competition on individual vs. population diets," Ecology, vol. 89, no. 7, pp. 1981-1993, 2008.

[42] T. Price, "Diet variation in a population of Darwin's finches," Ecology, vol. 68, no. 4, pp. 1015-1028, 1987.

[43] M. Doebeli and U. Dieckmann, "Evolutionary branching and sympatric speciation caused by different types of ecological interactions," American Naturalist, vol. 156, no. 4, pp. S77S101, 2000.

[44] R. J. Wootton, "The effect of size of food ration on egg production in the female three-spined stickleback, Gasterosteus aculeatus L," Journal of Fish Biology, vol. 5, no. 1, pp. 89-96, 1973.

[45] R. J. Wootton, "Effect of food limitation during the breeding season on the size, body components and egg production of female sticklebacks (Gasterosteus aculeatus)," Journal of Animal Ecology, vol. 46, pp. 823-834, 1977.

[46] R. J. Wootton, "Effects of food and density on the reproductive biology of the threespine stickleback with a hypothesis on population limitation in sticklebacks," Behaviour, vol. 93, pp. 101-111, 1985.

[47] T. Ingram, W. E. Stutz, and D. I. Bolnick, "Does intraspecific size variation in a predator affect its diet diversity and topdown control of prey?" PLOS One, vol. 6, Article ID e20782, 2011.

[48] D. I. Bolnick, "Can intraspecific competition drive disruptive selection? An experimental test in natural populations of sticklebacks," Evolution, vol. 58, no. 3, pp. 608-618, 2004. 
[49] S. M. Vamosi, T. Hatfield, and D. Schluter, "A test of ecological selection against young-of-the-year hybrids of sympatric sticklebacks," Journal of Fish Biology, vol. 57, no. 1, pp. 109-121, 2000.

[50] H. D. Rundle, S. M. Vamosi, and D. Schluter, "Experimental test of predation's effect on divergent selection during character displacement in sticklebacks," Proceedings of the National Academy of Sciences of the United States of America, vol. 100, no. 25, pp. 14943-14948, 2003.

[51] D. Schluter, "Experimental evidence that competition promotes divergence in adaptive radiation," Science, vol. 266, no. 5186, pp. 798-801, 1994.

[52] D. Schluter, "Frequency dependent natural selection during character displacement in sticklebacks," Evolution, vol. 57, no. 5, pp. 1142-1150, 2003.

[53] L. Snowberg, K. A. Hendrix, and D. I. Bolnick, "Individual diet specialization is widespread across lacustrine threespine stickleback," In preparation.

[54] D. I. Bolnick and J. Paull, "Diet similarity declines with morphological distance between conspecific individuals," Evolutionary Ecology Research, vol. 11, pp. 1217-1233, 2009.

[55] L. Snowberg, K. A. Hendrix, and D. I. Bolnick, "Variable variation: patterns of intraspecific niche variation across lake populations of threespine stickleback," In press.

[56] R. Svanbäck and D. I. Bolnick, "Intraspecific competition drives increased resource use diversity within a natural population," Proceedings of the Royal Society, vol. 274, no. 1611, pp. 839-844, 2007.

[57] D. I. Bolnick, E. J. Caldera, and B. Matthews, "Evidence for asymmetric migration load in a pair of ecologically divergent stickleback populations," Biological Journal of the Linnean Society, vol. 94, no. 2, pp. 273-287, 2008.

[58] B. Matthews, K. B. Marchinko, D. I. Bolnick, and A. Mazumder, "Specialization of trophic position and habitat use by sticklebacks in an adaptive radiation," Ecology, vol. 91, no. 4, pp. 1025-1034, 2010.

[59] B. W. Robinson, "Trade offs in habitat-specific foraging efficiency and the nascent adaptive divergence of sticklebacks in lakes," Behaviour, vol. 137, no. 7-8, pp. 865-888, 2000.

[60] D. Berner, R. Kaeuffer, A.-C. Grandchamp, J. A. M. Raeymaekers, K. Rasanen, and A. P. Hendry, "Quantitative genetic inheritance of morphological divergence in a lake-stream stickleback ecotype pair: implications for reproductive isolation," Journal of Evolutionary Biology, vol. 24, no. 9, pp. 19751983, 2011.

[61] T. Day, J. Pritchard, and D. Schluter, "A comparison of two sticklebacks," Evolution, vol. 48, no. 5, pp. 1723-1734, 1994.

[62] M. Hermida, C. Fernández, R. Amaro, and E. San Miguel, "Heritability and "evolvability" of meristic characters in a natural population of Gasterosteus aculeatus," Canadian Journal of Zoology, vol. 80, no. 3, pp. 532-541, 2002.

[63] C. L. Peichel, K. S. Nereng, K. A. Ohgi et al., "The genetic architecture of divergence between threespine stickleback species," Nature, vol. 414, no. 6866, pp. 901-905, 2001.

[64] S. M. Vamosi, "The presence of other fish species affects speciation in threespine sticklebacks," Evolutionary Ecology Research, vol. 5, no. 5, pp. 717-730, 2003.

[65] D. I. Bolnick, T. Ingram, W. E. Stutz, L. K. Snowberg, O. L. Lau, and J. S. Pauli, "Ecological release from interspecific competition leads to decoupled changes in population and individual niche width," Proceedings of the Royal Society B, vol. 277, no. 1689, pp. 1789-1797, 2010.
[66] J. W. Boughman, "Divergent sexual selection enhances reproductive isolation in sticklebacks," Nature, vol. 411, no. 6840, pp. 944-948, 2001.

[67] T. Hatfield and D. Schluter, "A test for sexual selection on hybrids of two sympatric sticklebacks," Evolution, vol. 50, no. 6, pp. 2429-2434, 1996.

[68] J. S. McKinnon, S. Mori, B. K. Blackman et al., "Evidence for ecology's role in speciation," Nature, vol. 429, no. 6989, pp. 294-298, 2004.

[69] H. D. Rundle and D. Schluter, "Reinforcement of stickleback mate preferences: sympatry breeds contempt," Evolution, vol. 52, no. 1, pp. 200-208, 1998.

[70] S. M. Vamosi and D. Schluter, "Sexual selection against hybrids between sympatric stickleback species: evidence from a field experiment," Evolution, vol. 53, no. 3, pp. 874879, 1999.

[71] C. Eizaguirre, T. L. Lenz, R. D. Sommerfeld, C. Harrod, M. Kalbe, and M. Milinski, "Parasite diversity, patterns of MHC II variation and olfactory based mate choice in diverging three-spined stickleback ecotypes," Evolutionary Ecology, vol. 25, no. 3, pp. 605-622, 2010.

[72] T. H. Vines and D. Schluter, "Strong assortative mating between allopatric sticklebacks as a by-product of adaptation to different environments," Proceedings of the Royal Society, vol. 273, no. 1589, pp. 911-916, 2006.

[73] J. A. M. Raeymaekers, M. Boisjoly, L. Delaire, D. Berner, K. Räsänen, and A. P. Hendry, "Testing for mating isolation between ecotypes: laboratory experiments with lake, stream and hybrid stickleback," Journal of Evolutionary Biology, vol. 23, no. 12, pp. 2694-2708, 2010.

[74] L. K. Snowberg and D. I. Bolnick, "Disentangling spatial segregation and assortative mating in threespine stickleback," Unpublished manuscript.

[75] A. J. W. Ward, P. J. B. Hart, and J. Krause, "The effects of habitat- and diet-based cues on association preferences in three-spined sticklebacks," Behavioral Ecology, vol. 15, no. 6, pp. 925-929, 2004.

[76] L. Snowberg and D. I. Bolnick, "Diet-dependent mate preferences in threespine stickleback," Unpublished manuscript.

[77] M. Milinski, "The function of mate choice in sticklebacks: optimizing Mhc genetics," Journal of Fish Biology, vol. 63, supplement A, pp. 1-16, 2003.

[78] M. A. Bell and S. A. Foster, The Evolutionary Biology of the Threespine Stickleback, Oxford University Press, New York, NY, USA, 1994.

[79] J. L. Gow, S. M. Rogers, M. Jackson, and D. Schluter, "Ecological predictions lead to the discovery of a benthiclimnetic sympatric species pair of threespine stickleback in Little Quarry Lake, British Columbia," Canadian Journal of Zoology, vol. 86, no. 6, pp. 564-571, 2008.

[80] J. S. McKinnon and H. D. Rundle, "Speciation in nature: the threespine stickleback model systems," Trends in Ecology and Evolution, vol. 17, no. 10, pp. 480-488, 2002.

[81] J. L. Gow, C. L. Peichel, and E. B. Taylor, "Ecological selection against hybrids in natural populations of sympatric threespine sticklebacks," Journal of Evolutionary Biology, vol. 20, no. 6, pp. 2173-2180, 2007.

[82] E. B. Taylor, J. W. Boughman, M. Groenenboom, M. Sniatynski, D. Schluter, and J. L. Gow, "Speciation in reverse: morphological and genetic evidence of the collapse of a three-spined stickleback (Gasterosteus aculeatus) species pair," Molecular Ecology, vol. 15, no. 2, pp. 343-355, 2006. 
[83] J. E. Behm, A. R. Ives, and J. W. Boughman, "Breakdown in postmating isolation and the collapse of a species pair through hybridization," American Naturalist, vol. 175, no. 1, pp. 11-26, 2010.

[84] W. A. Cresko and J. A. Baker, "Two morphotypes of lacustrine threespine stickleback, Gasterosteus aculeatus, in Benka Lake, Alaska," Environmental Biology of Fishes, vol. 45, no. 4, pp. 343-350, 1996.

[85] B. K. Kristjánsson, S. Skúlason, and D. L. G. Noakes, "Morphological segregation of Icelandic threespine stickleback (Gasterosteus aculeatus L)," Biological Journal of the Linnean Society, vol. 76, no. 2, pp. 247-257, 2002.

[86] B. K. Kristjánsson, S. Skúlason, and D. L. G. Noakes, "Rapid divergence in a recently isolated population of threespine stickleback (Gasterosteus aculeatus L.)," Evolutionary Ecology Research, vol. 4, no. 5, pp. 659-672, 2002.

[87] J. D. McPhail, "Ecology and evolution of sympatric sticklebacks (Gasterosteus): origin of the species pairs," Canadian Journal of Zoology, vol. 71, no. 3, pp. 515-523, 1993.

[88] E. B. Taylor and J. D. McPhail, "Evolutionary history of an adaptive radiation in species pairs of threespine sticklebacks (Gasterosteus): insights from mitochondrial DNA," Biological Journal of the Linnean Society, vol. 66, no. 3, pp. 271-291, 1999.

[89] H. D. Rundle, L. Nagel, J. W. Boughman, and D. Schluter, "Natural selection and parallel speciation in sympatric sticklebacks," Science, vol. 287, no. 5451, pp. 306-308, 2000.

[90] W. H. Mathews, J. G. Fyles, and H. W. Nasmith, "Postglacial crustal movements in southwestern British Columbia and adjacent Washington State," Canadian Journal of Earth Science, vol. 7, pp. 690-702, 1970.

[91] E. B. Taylor and J. D. McPhail, "Historical contingency and ecological determinism interact to prime speciation in sticklebacks, Gasterosteus," Proceedings of the Royal Society B, vol. 267, no. 1460, pp. 2375-2384, 2000.

[92] R. Kassen, D. Schluter, and J. D. McPhail, "Evolutionary history of threespine sticklebacks (Gasterosteus spp.) in British Columbia: insights from a physiological clock," Canadian Journal of Zoology, vol. 73, pp. 2154-2158, 1995.

[93] I. Hutchinson, T. S. James, J. J. Clague, J. V. Barrie, and K. W. Conway, "Reconstruction of late Quaternary sea-level change in southwestern British Columbia from sediments in isolation basins," Boreas, vol. 33, no. 3, pp. 183-194, 2004.

[94] D. I. Bolnick, "Waiting for sympatric speciation," Evolution, vol. 58, no. 4, pp. 895-899, 2004.

[95] M. Doebeli, H. J. Blok, O. Leimar, and U. Dieckmann, "Multimodal pattern formation in phenotype distributions of sexual populations," Proceedings of the Royal Society B, vol. 274, no. 1608, pp. 347-357, 2007.

[96] S. Gavrilets, "The Maynard Smith model of sympatric speciation," Journal of Theoretical Biology, vol. 239, no. 2, pp. 172-182, 2006.

[97] D. I. Bolnick and B. M. Fitzpatrick, "Sympatric speciation: models and empirical evidence," Annual Review of Ecology, Evolution, and Systematics, vol. 38, pp. 459-487, 2007.

[98] C. Matessi, A. Gimelfarb, and S. Gavrilets, "Long term buildup of reproductive isolation promoted by disruptive selection: how far does it go?" Selection, vol. 2, pp. 41-64, 2001.

[99] J. Polechová and N. H. Barton, "Speciation through competition: a critical review," Evolution, vol. 59, no. 6, pp. 1194 1210, 2005.
[100] A. M. Siepielski, J. D. Dibattista, and S. M. Carlson, "It's about time: the temporal dynamics of phenotypic selection in the wild," Ecology Letters, vol. 12, no. 11, pp. 1261-1276, 2009.

[101] A. M. Siepielski, J. D. Di Battista, J. A. Evans, and S. M. Carlson, "Differences in the temporal dynamics of phenotypic selection among fitness components in the wild," Proceedings of The Royal Society B, vol. 278, pp. 1572-1580, 2011.

[102] R. J. Wootton and C. Smith, "A long-term study of a short-lived fish: the demography of Gasterosteus aculeatus," Behaviour, vol. 137, no. 7-8, pp. 981-997, 2000.

[103] R. Svanbäck and L. Persson, "Population density fluctuations change the selection gradient in eurasian perch," American Naturalist, vol. 173, no. 4, pp. 507-516, 2009.

[104] T. E. Reimchen and P. Nosil, "Ecological causes of sex-biased parasitism in threespine stickleback," Biological Journal of the Linnean Society, vol. 73, no. 1, pp. 51-63, 2001.

[105] T. E. Reimchen and P. Nosil, "Temporal variation in divergent selection on spine number in threespine stickleback," Evolution, vol. 56, no. 12, pp. 2472-2483, 2002.

[106] T. E. Reimchen and P. Nosil, "Variable predation regimes predict the evolution of sexual dimorphism in a population of threespine stickleback," Evolution, vol. 58, no. 6, pp. 12741281, 2004.

[107] T. E. Reimchen and P. Nosil, "Replicated ecological landscapes and the evolution of morphological diversity among Gasterosteus populations from an archipelago on the west coast of Canada," Canadian Journal of Zoology, vol. 84, no. 5, pp. 643-654, 2006.

[108] J. Johansson and J. Ripa, "Will sympatric speciation fail due to stochastic competitive exclusion?" American Naturalist, vol. 168, no. 4, pp. 572-578, 2006.

[109] J. Johansson, J. Ripa, and N. Kuckländer, "The risk of competitive exclusion during evolutionary branching: effects of resource variability, correlation and autocorrelation," Theoretical Population Biology, vol. 77, no. 2, pp. 95-104, 2010.

[110] R. Svanbäck, M. Pineda-Krch, and M. Doebeli, "Fluctuating population dynamics promotes the evolution of phenotypic plasticity," American Naturalist, vol. 174, no. 2, pp. 176-189, 2009.

[111] M. Ackermann and M. Doebeli, "Evolution of niche width and adaptive diversification," Evolution, vol. 58, no. 12, pp. 2599-2612, 2004.

[112] J. Roughgarden, "Evolution of niche width," American Naturalist, vol. 106, pp. 683-718, 1972.

[113] M. Slatkin, "Frequency- and density-dependent selection on a quantitative character," Genetics, vol. 93, no. 3, pp. 755-771, 1979.

[114] C. Rueffler, T. J. M. Van Dooren, O. Leimar, and P. A. Abrams, "Disruptive selection and then what?" Trends in Ecology and Evolution, vol. 21, no. 5, pp. 238-245, 2006.

[115] D. I. Bolnick and M. Doebeli, "Sexual dimorphism and adaptive speciation: two sides of the same ecological coin," Evolution, vol. 57, no. 11, pp. 2433-2449, 2003.

[116] D. Berner, W. E. Stutz, and D. I. Bolnick, "Diversification in phenotypic (co)variances among lacustrine populations of threespine stickleback," Evolution, vol. 64, pp. 2265-2277, 2010.

[117] R. Shine, "Ecological causes for the evolution of sexual dimorphism: a review of the evidence," Quarterly Review of Biology, vol. 64, no. 4, pp. 419-461, 1989. 
[118] T. J. M. Van Dooren, M. Durinx, and I. Demon, "Sexual dimorphism or evolutionary branching?" Evolutionary Ecology Research, vol. 6, no. 6, pp. 857-871, 2004.

[119] I. Cooper, R. T. Gilman, and J. W. Boughman, "Sexual dimorphism and speciation on two ecological coins: patterns from nature and theoretical predictions," Evolution, vol. 65, no. 9, pp. 2553-2571, 2011.

[120] J. Kitano, S. Mori, and C. L. Peichel, "Sexual dimorphism in the external morphology of the threespine stickleback (Gasterosteus aculeatus)," Copeia, no. 2, pp. 336-349, 2007.

[121] T. Leinonen, J. M. Cano, and J. Merila, "Genetic basis of seual dimorphism in the threespine stickleback Gasterosteus aculeatus," Heredity, vol. 106, pp. 218-227, 2011.

[122] X. Thibert-Plante and A. Hendry, "The consequences of phenotypic plasticity for ecological speciation," Journal of Evolutionary Biology, vol. 24, pp. 326-342, 2011.

[123] D. W. Stephens and J. R. Krebs, Foraging Theory, Princeton University Press, Princeton, NJ, USA, 1986.

[124] E. E. Werner, "Optimal foraging and the size selection of prey by the bluegill sunfish (Lepomis macrochirus)," Ecology, vol. 55, pp. 1042-1052, 1974.

[125] R. Svanbäck and D. I. Bolnick, "Intraspecific competition affects the strength of individual specialization: an optimal diet theory method," Evolutionary Ecology Research, vol. 7, no. 7, pp. 993-1012, 2005.

[126] M. Dres and J. Mallet, "Host races in plant-feeding insects and their importance in sympatric speciation," Philosophical Transactions of the Royal Society of London B, vol. 357, pp. 471-472, 2002.

[127] M. Doebeli, U. Dieckmann, J. A. J. Metz, and D. Tautz, "What we have also learned: adaptive speciation is theoretically plausible," Evolution, vol. 59, no. 3, pp. 691-695, 2005.

[128] G. E. P. Box and N. R. Draper, Empirical Model Building and Response Surfaces, John Wiley \& Sons, Boston, Mass, USA, 1987.

[129] U. Dieckmann, M. Doebeli, J. A. J. Metz, and D. Tautz, Eds., Adaptive Speciation, Cambridge University Press, New York, NY, USA, 2004.

[130] R. Lande, "Models of speciation by sexual selection on polygenic traits," Proceedings of the National Academy of Sciences of the United States of America, vol. 78, no. 6, pp. 3721-3725, 1981.

[131] D. W. Hall, M. Kirkpatrick, and B. West, "Runaway sexual selection when female preferences are directly selected," Evolution, vol. 54, no. 6, pp. 1862-1869, 2000.

[132] R Development Core Team, R: A Language and Environment for Statistical Computing. $R$ Foundation for Statistical Computing, Vienna, Austria, 2007. 

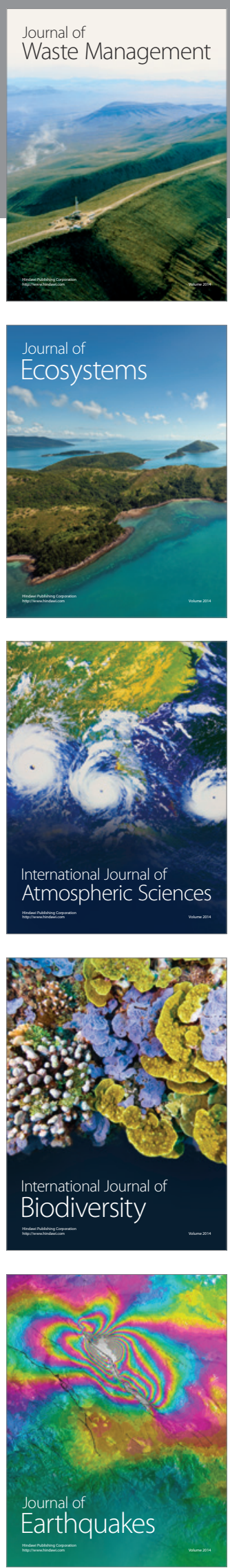
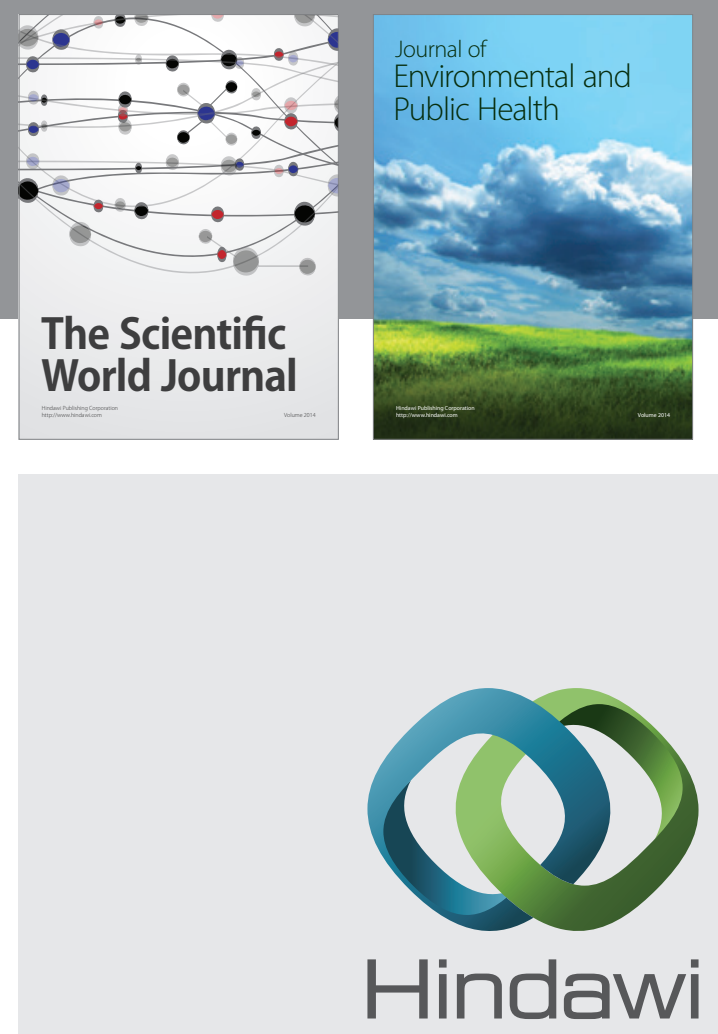

Submit your manuscripts at

http://www.hindawi.com
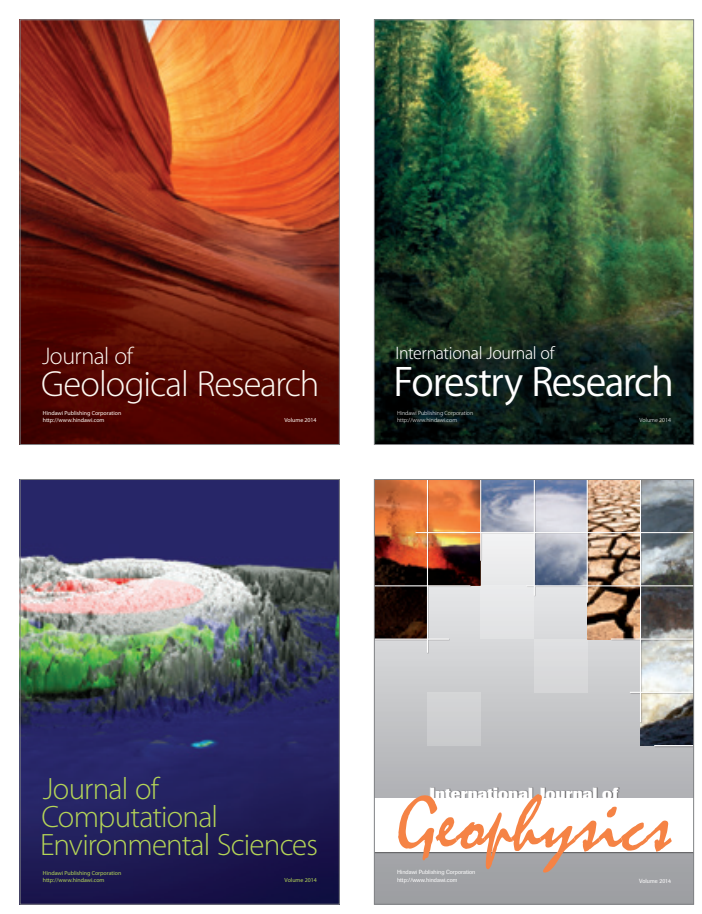
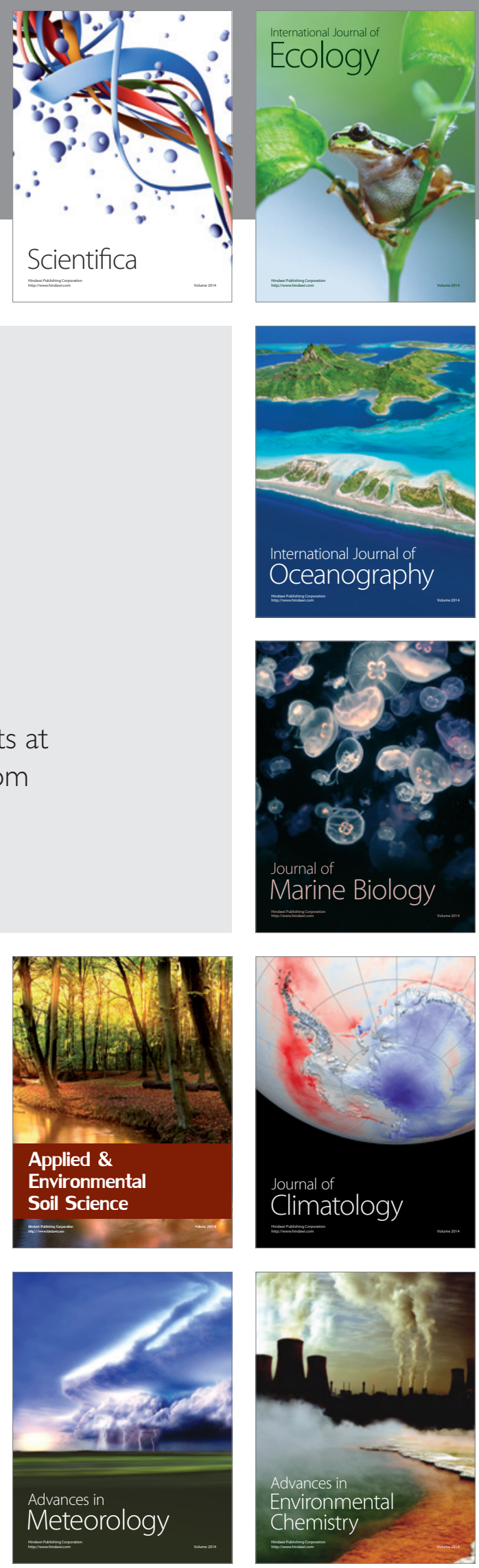